\title{
Effect of the herbal formulation Shen-Zhi-Ling on an APP/PS1 mouse model of Alzheimer's disease by modulating the biliverdin reductase/heme oxygenase 1 system
}

\author{
SANLI XING*, DINGZHU SHEN* , CHUAN CHEN, BEILING WU and HUIYING CHI \\ Geriatrics Laboratory, Shanghai Geriatric Institute of Chinese Medicine, \\ Shanghai University of Traditional Chinese Medicine, Shanghai 200031, P.R. China
}

Received June 15, 2016; Accepted April 7, 2017

DOI: $10.3892 /$ etm.2017.4732

\begin{abstract}
Shen-Zhi-Ling (SZL) oral liquid is a traditional Chinese medicine formula that is mainly used for the clinical treatment of mild to moderate Alzheimer's disease (AD). The aim of the present study was to investigate the effects and underlying mechanisms of SZL treatment on AD. APP/PS1 transgenic mice were utilized to evaluate the effect of SZL treatment $(0.5 \mathrm{~g} / 20 \mathrm{~g} / \mathrm{day})$. Morris water maze and Thioflavin $\mathrm{S}$ staining analyses were used to evaluate the cognitive impairment and $\beta$-amyloid plaques, respectively, while quantitative polymerase chain reaction and western blot analysis were performed to examine the mRNA and protein expression levels of heme oxygenase 1 (HO-1) and biliverdin reductase (BVR). Furthermore, immunofluorescence staining was used to measure the BVR and HO-1 protein levels in the hippocampus. The findings of the current study demonstrated that SZL treatment was able to ameliorate the impairment of memory and reduce the accumulation of amyloid plaques, and its ameliorating effects may be attributed to the modulation of the HO-1/BVR system in the hippocampus. These results indicate that SZL may be a possible complementary and alternative therapy to delay the development of AD.
\end{abstract}

\section{Introduction}

Alzheimer's disease (AD) is the most common type of dementia and it was estimated that $>44$ million people in 2015 had AD and this figure is expected to increase to 135 million

Correspondence to: Professor Chuan Chen, Geriatrics Laboratory, Shanghai Geriatric Institute of Chinese Medicine, Shanghai University of Traditional Chinese Medicine, 365C Xiangyang South Road, Shanghai 200031, P.R. China

E-mail: article_chenchuan@163.com

${ }^{*}$ Contributed equally

Key words: Alzheimer's disease, Shen-Zhi-Ling oral liquid, heme oxygenase 1 , biliverdin reductase by 2050 throughout the world (1). Neuropathological studies have confirmed that extracellular senile plaque deposition, intracellular nerve fiber tangles and neuronal degeneration loss are the main pathological features of AD (2-4). With the population aging, AD is predicted to become an important worldwide disease threatening human health.

The US Food and Drug Administration have approved five drugs, including donepezil, memantine, galantamine, rivastigmine and tacrine, for the treatment of $\mathrm{AD}$; however, these only provide mild symptomatic relief and do not prevent the development of dementia (5). Therefore, there is a requirement for alternative drugs for the treatment of AD. In addition, accumulating studies have recently demonstrated that herbal preparations may provide a prospective alternative in the treatment of dementia due to better compliance and reduced side effects (6,7). Shen-Zhi-Ling (SZL) oral liquid is China's first approved compound of traditional Chinese medicine (TCM) for mild to moderate AD. Previous evidence from clinical and experimental studies has demonstrated that SZL can improve the cognitive function of patients with mild to moderate AD (8), and raise the brain function by increasing the connectivity between posterior cingulated gyrus and specific areas in the brain (9). However, the exact mechanism underlying SZL-mediated cognitive improvements remains unknown. Collectively, these earlier studies suggest that the beneficial effect of SZL concoction involves multiple targets and pathways. Therefore, the present study aimed to identify target proteins of the $S Z L$ concoction that were particularly associated with the treatment of AD.

\section{Materials and methods}

Ethics statement. All procedures were performed in accordance with the Guide for the Care and Use of Medical Laboratory Animals (Ministry of Health of China, 1998) and the guidelines of the Shanghai University of Traditional Chinese Medicine (Shanghai, China) Laboratory Animal Care and Use Committee. Animal suffering and discomfort were minimized.

APP/PS1 transgenic mice and drug treatment. In total, 20 male $\mathrm{APP}_{\mathrm{swe}} / \mathrm{PS}_{\Delta \mathrm{E} 9}(\mathrm{APP} / \mathrm{PS} 1)$ transgenic mice (age, 
6 months; weight, $26 \pm 3 \mathrm{~g}$ ) and 10 male C57BL/6 mice (age, 6 months; (weight, $27 \pm 2 \mathrm{~g}$ ) were used in the present study. The APP/PS1 mouse strain (10) is a double-transgenic hemizygote that expresses a chimeric mouse/human amyloid precursor protein and mutant human presenilin-1. The transgenic mice were used as they develop behavioral and pathology features of AD similar to patients and the mice have been widely used in AD studies worldwide (11). C57BL/6 mice were used as age-matched controls and APP/PS1 transgenic mice were purchased from the Model Animal Research Center of Nanjing University (Nanjing, China), while C57BL/6 mice were obtained from the Experimental Animal Center of Shanghai Academy (Shanghai, China). The mice were housed in a controlled specific-pathogen-free environment $\left(22 \pm 3^{\circ} \mathrm{C}\right.$; $60 \%$ relative humidity; and access to a 12 -h light/dark cycle) and ad libitum access to water and food.

SZL, which is the TCM formula used in this study, is an oral liquid consisting of 10 types of TCM components $(10 \mathrm{ml}$ each; $74.5 \mathrm{~g}$ crude drug), as follows: Codonopsis pilosula, Cassia Twig, Paeonia lactiflora, honey-fried Licorice root, Poria cocos, Rhizoma Zingiberis, Radix Polygalae, Acorus tatarinowii, Ossa Draconis and Concha Ostreae. Detailed extraction and quality control information was obtained from the manufacturer (Shandong Wohua Pharmaceuticals Co., Ltd., Weifang, China). Mice were divided into three groups, with 10 mice per group, as follows: C57BL/6 (control), APP/PS1, and APP/PS1+SZL groups. The AD model group (APP/PS1 group) contained APP/PS1 mice that received no treatment. The APP/PS1+SZL group consisted of APP/PS1 transgenic mice that received $200 \mu \mathrm{l}$ SZL $(2.5 \mathrm{~g} / \mathrm{ml})$ per $20 \mathrm{~g}$ mouse body weight by gavage administration once per day for 12 weeks. The male C57BL/6 mice group (control) was included as a normal control.

Morris water maze (MWM) test. The MWM test was performed as previously described, with minor modifications (12). The water maze $(120 \mathrm{~cm}$ in diameter) used was filled with water, and a hidden platform was submerged $1 \mathrm{~cm}$ beneath the water surface. The water temperature was kept between 20 and $23^{\circ} \mathrm{C}$. Each mouse was trained to locate the hidden platform in four trials per day with an inter-trial interval of 30-40 min, which continued for 5 days. The mouse was allowed to search for the platform for $\leq 70 \mathrm{sec}$, allowing it to rest $30 \mathrm{sec}$ on the platform. The time the mice spent finding the platform was recorded as the escape latency. On day 6 , the platform was removed for a $70 \mathrm{sec}$ probe trial, during which the time spent in each quadrant and number of platform crosses was recorded. The experiments were recorded with a camera connected to a video recorder and a computerized tracking system.

Thioflavin S staining. Mice were anesthetized with 5\% chloral hydrate, and saline solution was used for perfusion through the heart, which was followed by $4 \%$ paraformaldehyde. The brains of the mice were then removed, fixed in $4 \%$ paraformaldehyde for $24 \mathrm{~h}$ and immersed in $30 \%$ sucrose until they sank. Next, $30-\mu \mathrm{m}$ coronal floating sections were immersed in 1\% Thioflavin S solution (Sigma-Aldrich; Merck, Darmstadt, Germany) for 9 min and differentiated in 70\% alcohol for 5 min. Subsequently, sections were washed in 80 and $95 \%$ ethanol, followed by three washes with $\mathrm{ddH}_{2} \mathrm{O}$, and were then mounted. Fluorescent signals were detected by fluorescence microscopy (Olympus BX51; Olympus Corp., Tokyo, Japan).

Reverse transcription-quantitative polymerase chain reaction $(R T-q P C R)$. Anesthetized animals were sacrificed, and hippocampus tissues were immediately frozen in liquid nitrogen. Total RNA was extracted using TRIzol reagent (Invitrogen; Thermo Fisher Scientific, Inc., Waltham, MA, USA) according to the manufacturer's instructions. RNA purity and concentration was confirmed by measuring the ratio of absorbance at 260 and $280 \mathrm{~nm}$ using a spectrophotometer. Next, $2 \mu \mathrm{g}$ RNA was processed for cDNA synthesis using an RT system (A6010; Promega Corp., Madison, WI, USA). The primers used for RT-qPCR were the following: HO-1 forward, 5'-AAGCCG AGAATGCTGAGTTCA-3', and reverse, 5'-GCCGTGTAG ATATGGTACAAGGA-3'; BVR forward, 5'-CCTGATTGG ATATGTGTCCAGAC-3', and reverse, 5'-CGACATCAACCT CTTGGCTG-3'; GADPH forward, 5'-CATCCACTGGTG CTGCCAAGGCTGT-3', and reverse, 5'-ACAACCTGGTCC TCAGTGTAGCCCA-3'. The mRNA levels of GADPH were used as normalization controls. The reaction system included: $10 \mu \mathrm{l}$ PCR mix, $5 \mu \mathrm{l}$ subgreen mix, $1 \mu \mathrm{l}$ each of upstream and downstream primers and $3 \mu \mathrm{l}$ cDNA template to a total volume of $20 \mu \mathrm{l}$. The cycling parameters for all assays were as follows: $2 \mathrm{~min}$ at $50^{\circ} \mathrm{C}, 10 \mathrm{~min}$ at $95^{\circ} \mathrm{C}$, followed by 40 cycles of $15 \mathrm{sec}$ at $95^{\circ} \mathrm{C}$ and $1 \mathrm{~min}$ at $60^{\circ} \mathrm{C}$. The $2^{-\Delta \Delta \mathrm{Cq}}$ relative quantitative method was used to show the relative expression levels of each target gene (13).

Western blot analysis. The isolated hippocampi were collected and homogenized in lysis buffer (Beyotime Biotechnology, Inc., Shanghai, China), schizolysised for $30 \mathrm{~min}$ on ice and centrifuged at $12,000 \mathrm{x}$ g for $20 \mathrm{~min}$ at $4^{\circ} \mathrm{C}$. Total protein concentration was determined using a Micro BCA ${ }^{\mathrm{TM}}$ Protein Assay kit (23235; Pierce; Thermo Fisher Scientific, Inc.). A total of $30 \mathrm{mg}$ protein samples were heated at $95^{\circ} \mathrm{C}$ for $5 \mathrm{~min}$ and separated by $10 \%$ SDS-PAGE. Protein samples in the gel were transferred onto polyvinylidene fluoride membranes and membranes were blocked for $1 \mathrm{~h}$ in Tris-buffered saline/Tween-20 buffer (TBST; Beyotime Institute of Biotechnology, Haimen, China) containing 5\% bovine serum. Following blocking, the membranes were incubated with anti-BVR (1:5,000; ab19260; Abcam, Cambridge, MA, USA), anti-HO-1 (1:2,000; ab13243; Abcam) or anti- $\beta$-actin $\left(1: 5,000\right.$; ab8226; Abcam) primary antibodies at $4^{\circ} \mathrm{C}$ overnight. The membranes were washed with TBST three times every $10 \mathrm{~min}$ and then treated with goat anti-mouse or goat anti-rabbit IR-800 cw labeled secondary antibodies (926-32210 and 926-32211; LI-COR Biosciences, Inc., Lincoln, NE, USA) for $1 \mathrm{~h}$ at room temperature. Blots were washed three times for $10 \mathrm{~min}$ in TBST and membranes were imaged using an Odyssey ${ }^{\circledR}$ scanner and Odyssey ${ }^{\circledR}$ imaging software 3.0 (LI-COR Biosciences, Inc). Scan settings were medium or high image quality, $169 \mu \mathrm{m}$ resolution, intensity 5.0 for the 800-channel. Quantification of the proteins of interest was determined relative to $\beta$-actin using Image $\mathbf{J}$ software (National Institutes of Health- Bethesda, MD, USA).

Immunofluorescence analysis. Sections were permeabilized with $0.2 \%$ Triton $\mathrm{X}-100$, and then blocked with blocking 
solution containing 5\% normal serum bovine serum at room temperature for $30 \mathrm{~min}$. Next, sections were incubated with primary antibodies overnight at $4^{\circ} \mathrm{C}$, including polyclonal anti-BVR (1:1,000; ab19260; Abcam) and polyclonal anti-HO-1 (1:200; ab13243; Abcam). Subsequent to rinsing with phosphate-buffered saline (PBS), sections were further treated with fluorescein isothiocyanate (1:200; sc-2359; Santa Cruz Biotechnology, Inc., Dallas, TX, USA) secondary antibodies for $1 \mathrm{~h}$ at $37^{\circ} \mathrm{C}$. After three further washes in PBS, the sections were stained for nuclei with $5 \mu \mathrm{g} / \mathrm{ml}$ DAPI for $10 \mathrm{~min}$ at room temperature, and then mounted on glass slides. Fluorescent signals were detected by fluorescence microscopy.

Statistical analysis. Statistical analysis was conducted using GraphPad Prism version 5 software (GraphPad Software, Inc., La Jolla, CA, USA). Measurement data are presented as the mean + SEM (standard error of the mean). One-way ANOVA was used to assess multiple groups of data and Student's t-test was used to compare between two groups. $\mathrm{P}<0.05$ was considered to indicate a statistically significant difference.

\section{Results}

$S Z L$ reverses the memory impairment in APP/PS1 mice. Memory impairment in APP/PS1 transgenic mice was determined using the MWM test, in terms of the escape latency duration. As shown in Fig. 1A, the average escape latencies were significantly prolonged in the APP/PS1 group from the third to the fifth training day compared with the control group $(\mathrm{P}<0.01)$. After administering SZL oral liquid, the APP/PS1 group demonstrated a significant decrease in the escape latency compared with that of the APP/PS1 group without any treatment $(\mathrm{P}<0.05)$. There was a significant difference in escape latency between the APP+SZL and control group $(\mathrm{P}<0.01)$. On day 6 of the MWM test, the hidden platform was removed from the pool, and all groups of mice were allowed to swim for $70 \mathrm{sec}$. As shown in Fig. 1B, in the target quadrant on day 6, the APP/PS1 group spent significantly less time in the quadrant containing the platform in comparison with that in the control group $(\mathrm{P}<0.05)$. In addition, the mice in the SZL-treated group spent an increased amount of time in the target quadrant when compared with the untreated APP/PS1 group $(\mathrm{P}<0.05$; Fig. 1B). There was no significant difference in time in the target quadrant between the APP+SZL and control group. Furthermore, the number of times the platform was crossed was significantly reduced in the APP/PS1 group compared with the C57 control group $(\mathrm{P}<0.01)$ and the mice with SZL treatment group also exhibited a significantly increased number of platform crosses compared with the APP/PS1 group $(\mathrm{P}<0.05$; Fig. $1 \mathrm{C})$. There was no significant difference in number of platform crosses between APP/PS1 mice with SZL treatment and control mice. The results from the MWM tests indicated that SZL improved the spatial learning and memory deficits in AD mice.

SZL reduces amyloid pathology in APP/PS1 mice. In order to assess the effect of SZL treatment on AD neuropathology, staining with Thioflavin $\mathrm{S}$ for amyloid plaques was conducted. As expected, the AD mice presented numerous plaques at 9 months of age as compared with the controls; however, the
A

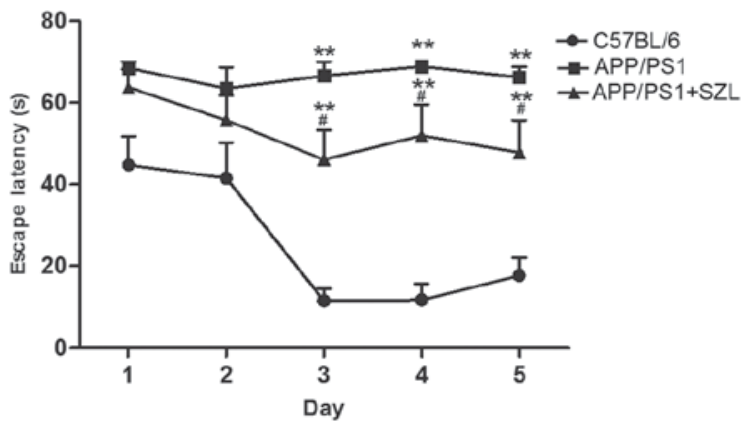

B

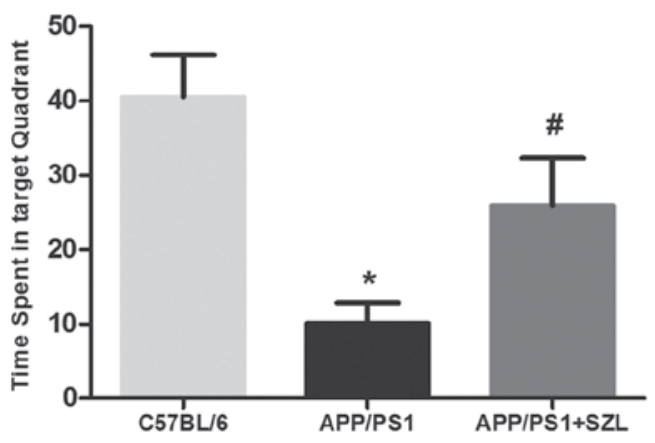

C

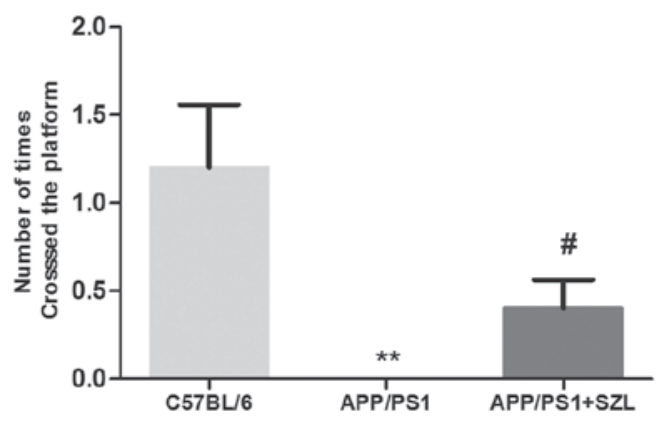

Figure 1. APP/PS1 mice exhibit decreased performance in the Morris water maze task, which is improved by SZL treatment. (A) Escape latency (time required to find the hidden platform) was determined in 5-day trial study. (B) Time spent in the target quadrant and (C) the number of platform crosses were determined on day 6 (probe trial; total time of the trial, $70 \mathrm{sec}$ ). Data are expressed the mean + standard error $(n=10)$. ${ }^{*} \mathrm{P}<0.05$ and ${ }^{* *} \mathrm{P}<0.01$ vs. C57BL/6 group; ${ }^{*} \mathrm{P}<0.05$ vs. APP/PS1 group. SZL, Shen-Zhi-Ling.

amyloid plaque presence was notably decreased in the hippocampus of mice treated with SZL (Fig. 2).

Effect of SZL treatment on mRNA levels of BVR and HO-I in the hippocampus of mice. To confirm the effect of SZL treatment on oxidative stress-associated molecules, RT-qPCR analysis was performed to determine BVR and HO-1 mRNA expression levels. As previously reported, the BVR/HO-1 system in APP/PS1 transgenic mice was impaired (14); thus, in the present study, mice were treated with SZL to determine whether treatment was able to directly modulate the BVR/HO-1 system. The results shown in Fig. 3 revealed that BVR and HO-1 mRNA levels in the APP/PS1 group were significantly decreased as compared with those in the control group $(\mathrm{P}<0.01)$. However, SZL treatment significantly increased the BVR and HO-1 levels compared with the untreated APP/PS1 mice (Fig. 3). There was a significant difference only in BVR 

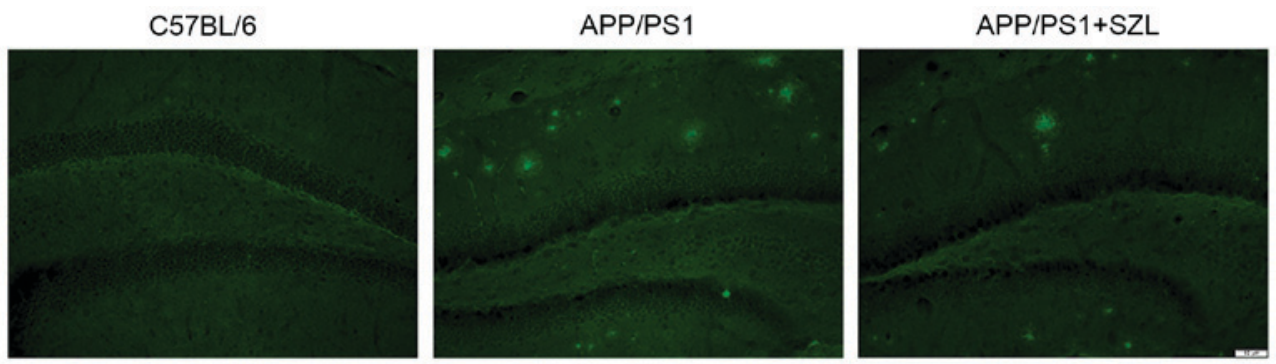

Figure 2. Histological examples of Thioflavin S staining in the hippocampus and SZL treatment can reduce the number of amyloid plaques. SZL, Shen-Zhi-Ling.

mRNA expression levels between the APP/PS1+SZL and control group $(\mathrm{P}<0.01)$, but no significant difference in $\mathrm{HO}-1$ mRNA expression level.

Effect of SZL treatment on protein levels of BVR and HO-1 in the hippocampus of mice. Given that SZL had significant effects on the mRNA expression levels of BVR and HO-1, protein extracts were prepared and analyzed by western blot analysis to also determine their protein levels. As shown in Fig. 4, consistent with the changes in mRNA levels, the protein expression of BVR and HO-1 in SZL-treated mice was significantly increased when compared with the APP/PS1 group $(\mathrm{P}<0.05)$. The only significant difference was observed in BVR protein expression levels between the APP+SZL and control group $(\mathrm{P}<0.01)$. In order to confirm the overexpression of BVR and HO-1 proteins in the hippocampus of APP/PS1 transgenic mice and the effect of SZL treatment, immunofluorescence analysis was then performed. It was observed that the hippocampi of APP/PS1 transgenic mice exhibited a reduced amount of immunoreactive BVR (Fig. 5A) and HO-1 (Fig. 5B) cells compared with the control mice, while SZL treatment reversed this decrease. These results indicated that the HO-1/BVR system serve an important role in the effect of SZL treatment on AD.

\section{Discussion}

Numerous studies on AD during the past decade have confirmed the involvement of oxidative stress in disease pathogenesis $(15,16)$. Oxidative stress involves the production of reactive oxygen species that exceeds the capability of the antioxidant systems (17). Therefore, increasing or protecting the antioxidative defense system from being attacked may be responsible for the decreasing memory caused by oxidative products, which are increased in AD patients when compared with controls (18). As Barone et al (19) previously demonstrated, an impairment of the HO-1/BVR system was detected in the hippocampus of AD subjects, and oxidative stress was observed in the accorded brain area. BVR is able to convert biliverdin (BV) into bilirubin (BR), and BR protects cells against oxidative stress. Subsequent amplifies the oxidation of BR by hydrogen peroxide back into $\mathrm{BV}$, which is then recycled back into BR by BVR (20). Thus upregulating the HO-1/BVR system was proposed as a useful mechanism to counteract AD-induced oxidative damage $(21,22)$.

The present study identified that BVR protein levels were decreased in the hippocampus of APP/PS1 mice (age, 9 months), compared with the wild-type controls. To further

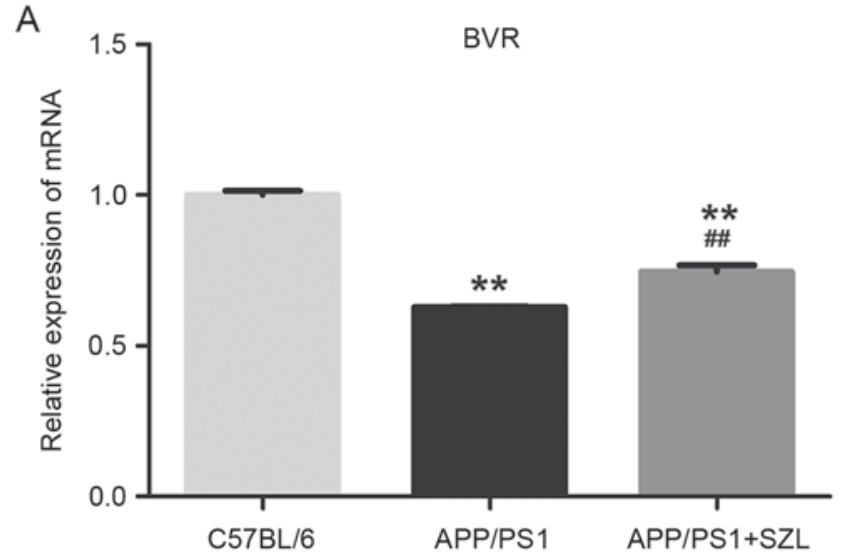

B

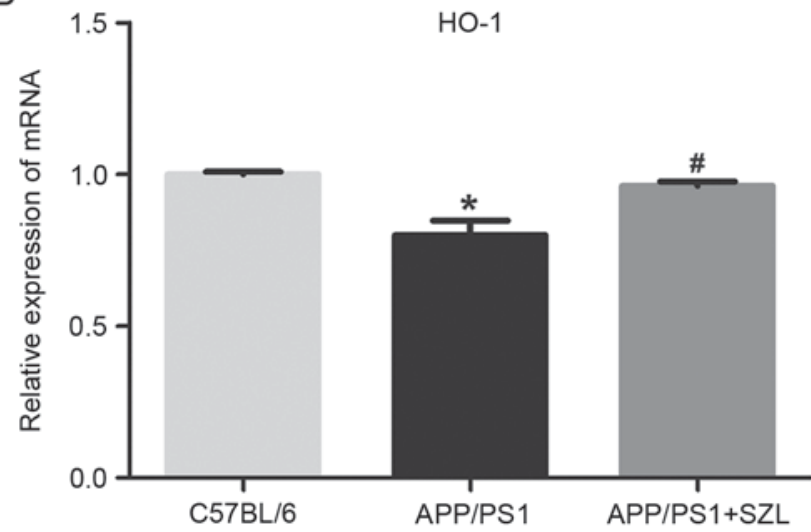

Figure 3. Effects of SZL treatment on mRNA expression levels of (A) BVR and (B) HO-1 in the hippocampus of mice after 12 weeks of treatment, determined by reverse transcription-quantitative polymerase chain reaction. Values are demonstrated as the mean + standard error. ${ }^{*} \mathrm{P}<0.05$ and ${ }^{* * *} \mathrm{P}<0.01$ vs. $\mathrm{C} 57 \mathrm{BL} / 6$ group; ${ }^{\#} \mathrm{P}<0.05$ and ${ }^{\# \#} \mathrm{P}<0.01$ vs. APP/PS1 group. SZL, Shen-Zhi-Ling; BVR, biliverdin reductase; HO-1, heme oxygenase 1.

understand whether the reduction of BVR protein levels was a result of antioxidant system not being active, the expression of its upstream associated protein HO-1 was also evaluated, which would be activated as a response to oxidative stress induction. As expected, HO-1 protein was consistently altered along with the BVR levels. According to these finding of the current study, the neurobiological benefits of BVR in the brain may initially include production of the powerful antioxidant BR as result of its reductase activity (23), followed by BVR regulating HO-1 expression, which forms a powerful protective system against oxidative stresses $(24,25)$. In addition, a possible 

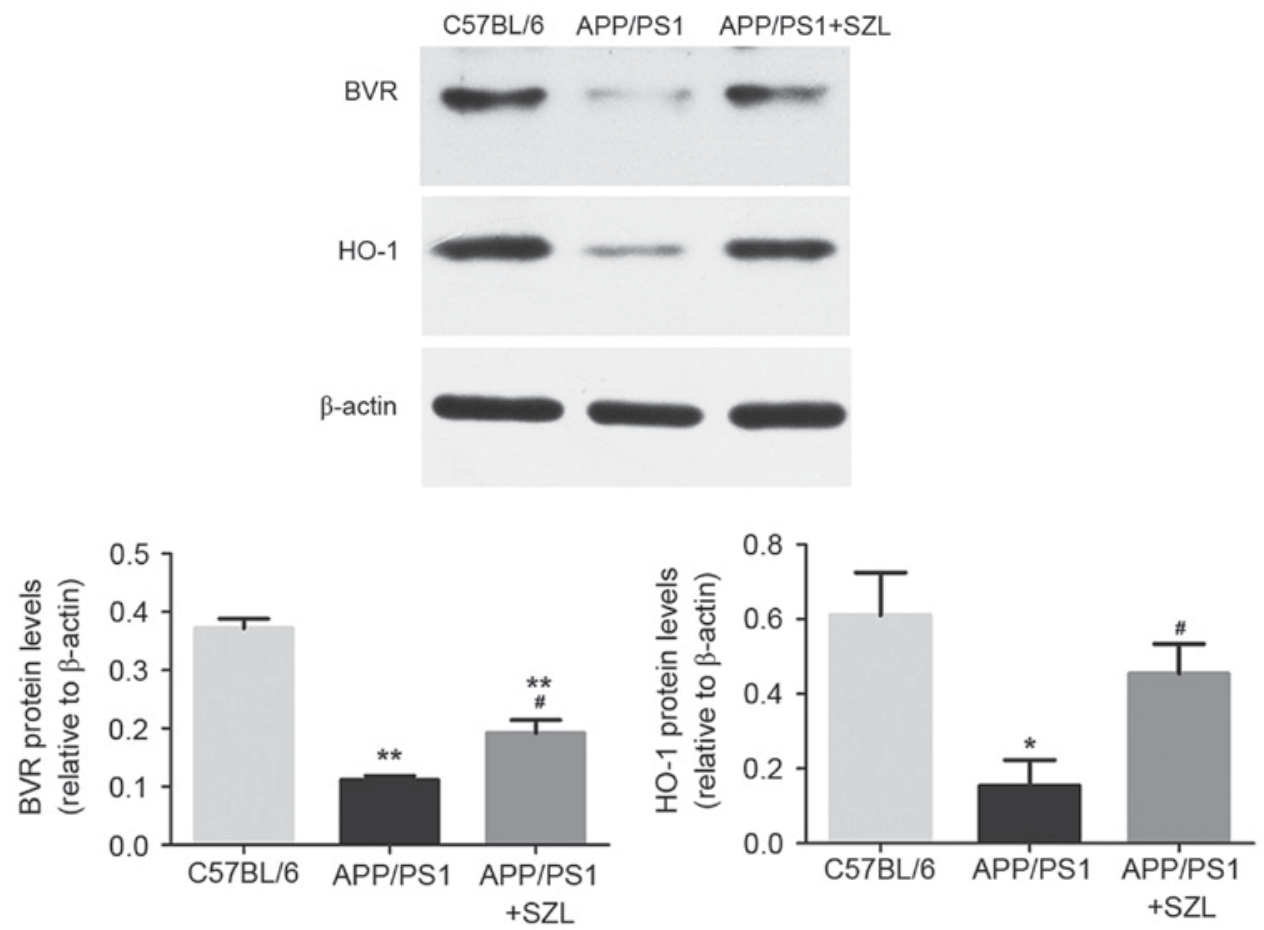

Figure 4. Western blot analyses of BVR and HO-1 levels in the hippocampus homogenates from 9-month-old mice. "P<0.05 and ${ }^{* *} \mathrm{P}<0.01 \mathrm{vs.}$ C57BL/6 group; ${ }^{\#} \mathrm{P}<0.05$ vs. APP/PS1 group. SZL, Shen-Zhi-Ling; BVR, biliverdin reductase; HO-1, heme oxygenase 1.

A
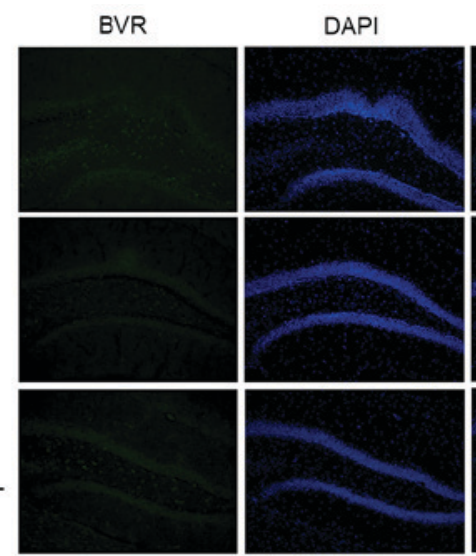

B
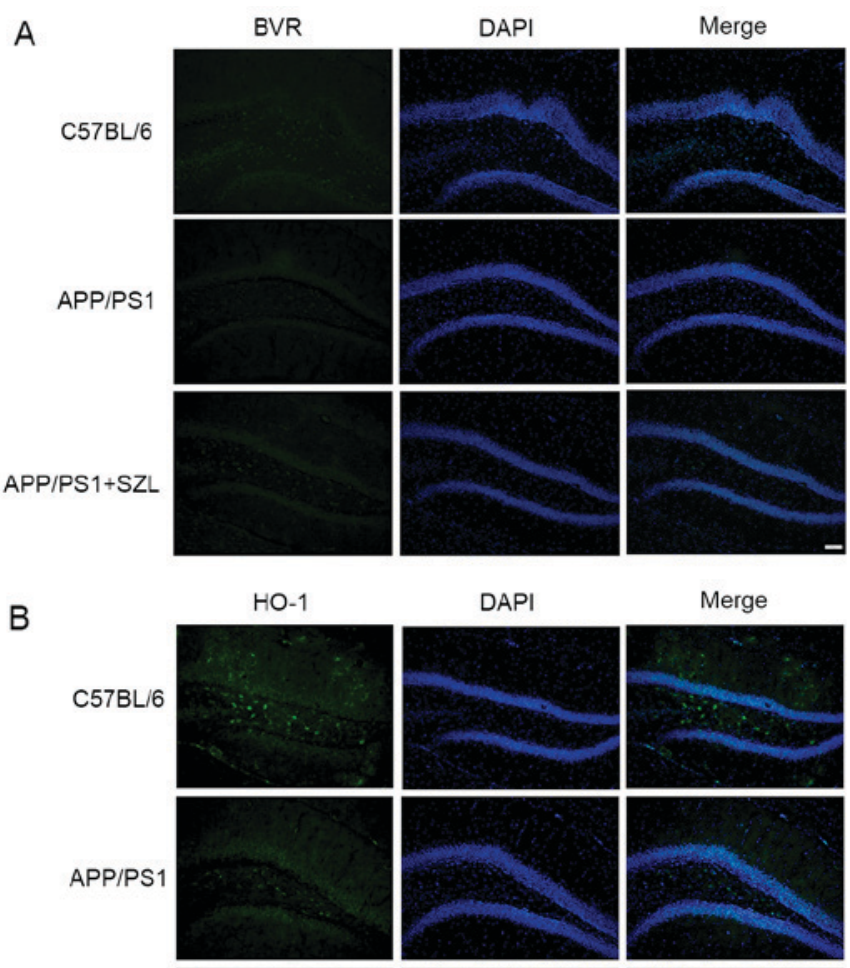

APP/PS1+SZL
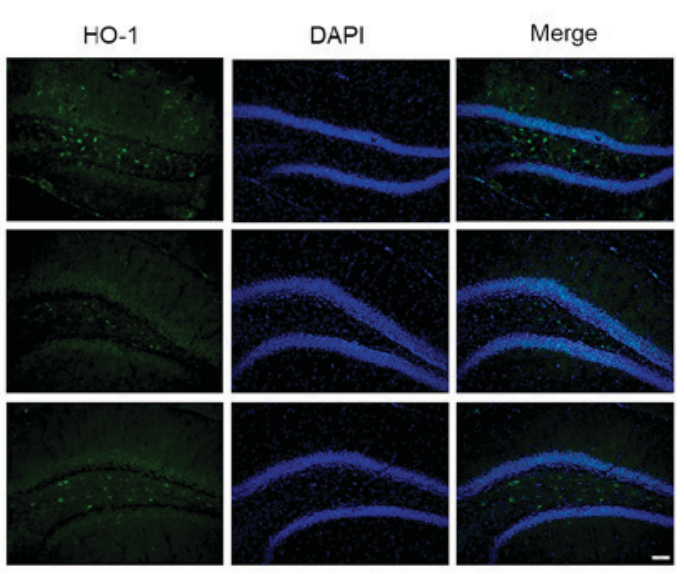

Figure 5. Effect of SZL treatment on the expression of (A) BVR and (B) HO-1, detected by immunofluorescence staining. Representative photomicrograph was shown. SZL, Shen-Zhi-Ling; BVR, biliverdin reductase; HO-1, heme oxygenase 1 . interaction between BVR and amyloid plaques production is proposed, but further experiments are required to confirm this. Furthermore, BVR not only reduces BV into BR, but is also a serine/threonine/tyrosine kinase involved in various cellular functions $(20,26,27)$. As previous study reported, increasing BVR protein may trigger a cell stress response and protect the cell from oxidant attack $(20,28)$, while the upregulation of the HO-1/BVR system has been proposed as a useful mechanism to counteract $\mathrm{AD}$-induced oxidative damage. Taken together, these findings indicate that increasing HO-1/BVR expression may have a therapeutic effect in AD.

TCM has a long history of preventing and treating cognitive decline (29) and the efficacy of Chinese herbs to improve cognitive function has been studied in various trials (30). SZL, as a drug approved by the China Food and Drug Administration for treatment or prevention of mild to moderate $\mathrm{AD}$, can ameliorate $\mathrm{AD}$-associated pathological symptoms. Among the ten components of SZL, Codonopsis pilosula may enhance intelligence. Radix Polygalae, Acorus tatarinowii and Poria cocos have been indicated to enhance cognitive function (31-33), whereas Ossa Draconis and Concha Ostreae are well-known sedatives, and studies in various countries have demonstrated that they have a sleep-inducing function for treating sleep disorders (34-36). Finally, Paeonia lactiflora and honey-fried Licorice root ate Paeonia lactiflora and honey-fried Licorice root are known to increase blood circulation in the brain (37).

In conclusion, SZL treatment in the present study was found to ameliorate AD-associated symptoms, as well as to reduce $A \beta$ amyloidosis and its ameliorating effects may be attributed to the modulation of the HO-1/BVR system in the hippocampus. SZL treatment can increase the expression of BVR and HO-1 protein, with a more effective increase in HO-1 expression. However, further investigation is required 
to identify the active functional constituents in SZL, as well as to elucidate the underlying molecular mechanisms. In addition, the exact mechanism by which SZL increases HO-1/BVR protein levels was not elucidated in the current study, thus additional experiments are required.

\section{Acknowledgements}

The present study was supported by grants from the National Natural Science Foundation of China (grant no. 81503626), the Shanghai Nature Science Fund (grant no. 13ZR1439800) and the Shanghai Health Bureau Youth Fund (grant no. 201540254).

\section{References}

1. Van Cauwenberghe C, Van Broeckhoven C and Sleegers K: The genetic landscape of Alzheimer disease: Clinical implications and perspectives. Genet Med 18: 421-430, 2015.

2. Perl DP: Neuropathology of Alzheimer's disease. Mt Sinai J Med 77: 32-42, 2010.

3. Ingelsson M, Fukumoto $\mathrm{H}$, Newell KL, Growdon JH, Hedley-Whyte ET, Frosch MP, Albert MS, Hyman BT and Irizarry MC: Early Abeta accumulation and progressive synaptic loss, gliosis, and tangle formation in AD brain. Neurology 62: 925-931, 2004

4. Karran E, Mercken M and De Strooper B: The amyloid cascade hypothesis for Alzheimer's disease: An appraisal for the development of therapeutics. Nat Rev Drug Discov 10: 698-712, 2011.

5. Winslow BT, Onysko MK, Stob CM and Hazlewood KA: Treatment of Alzheimer disease. Am Fam Physician 83: 1403-1412, 2011.

6. Hou Y, Wang Y, Zhao J, Li X, Cui J, Ding J, Wang Y, Zeng X, Ling Y, Shen X, et al: Smart Soup, a traditional Chinese medicine formula, ameliorates amyloid pathology and related cognitive deficits. PLoS One 9: e111215, 2014.

7. Durairajan SS, Huang YY, Yuen PY, Chen LL, Kwok KY, Liu LF, Song JX, Han QB, Xue L, Chung SK, et al: Effects of Huanglian-Jie-Du-Tang and its modified formula on the modulation of amyloid- $\beta$ precursor protein processing in Alzheimer's disease models. PLoS One 9: e92954, 2014.

8. Pan W, Wang Q, Kwak S, Song Y, Qin B, Wang M and Yamamoto Y: Shen-zhi-ling oral liquid improves behavioral and psychological symptoms of dementia in Alzheimer's disease. Evid Based Complement Alternat Med 2014: 913687, 2014.

9. Yu L, Lin SM, Zhou RQ, Tang WJ, Huang PX, Dong Y, Wang J, $\mathrm{Yu} \mathrm{ZH}$, Chen JL, Wei L, et al: Chinese herbal medicine for patients with mild to moderate Alzheimer disease based on syndrome differentiation: A randomized controlled trial. Zhong Xi Yi Jie He Xue Bao 10: 766-776, 2012 (In Chinese).

10. Borchelt DR, Ratovitski T, van Lare J, Lee MK, Gonzales V, Jenkins NA, Copeland NG, Price DL and Sisodia SS: Accelerated amyloid deposition in the brains of transgenic mice coexpressing mutant presenilin 1 and amyloid precursor proteins. Neuron 19: 939-945, 1997.

11. Liu H, Tian T, Qin S, Li W, Zhang X, Wang X, Gao Y and Huang G: Folic acid deficiency enhances abeta accumulation in APP/PS1 mice brain and decreases amyloid-associated miRNAs expression. J Nutr Biochem 26: 1502-1508, 2015.

12. D'Souza Y, Elharram A, Soon-Shiong R, Andrew RD and Bennett BM: Characterization of Aldh2 (-/-) mice as an age-related model of cognitive impairment and Alzheimer's disease. Mol Brain 8: 27, 2015.

13. Liu Z, Li S, Liu L, Guo Z and Wang P: Internal associations and dynamic expression of c-kit and nanog genes in ventricular remodelling induced by adriamycin. Exp Ther Med 12: $1657-1662,2016$.

14. Barone E, Di Domenico F, Cenini G, Sultana R, Coccia R, Preziosi P, Perluigi M, Mancuso C and Butterfield DA: Oxidative and nitrosative modifications of biliverdin reductase-A in the brain of subjects with Alzheimer's disease and amnestic mild cognitive impairment. J Alzheimers Dis 25: 623-633, 2011

15. Padurariu M, Ciobica A, Lefter R, Serban IL, Stefanescu C and Chirita R: The oxidative stress hypothesis in Alzheimer's disease. Psychiatr Danub 25: 401-409, 2013.
16. Kosenko EA, Solomadin IN, Tikhonova LA, Reddy VP, Aliev G and Kaminsky YG: Pathogenesis of Alzheimer disease: Role of oxidative stress, amyloid- $\beta$ peptides, systemic ammonia and erythrocyte energy metabolism. CNS Neurol Disord Drug Targets 13: 112-119, 2014.

17. Poon HF, Calabrese V, Scapagnini G and Butterfield DA: Free radicals and brain aging. Clin Geriatr Med 20: 329-359, 2004.

18. Perrig WJ, Perrig P and Stähelin HB: The relation between antioxidants and memory performance in the old and very old. J Am Geriatr Soc 45: 718-724, 1997.

19. Barone E, Di Domenico F, Sultana R, Coccia R, Mancuso C, Perluigi M and Butterfield DA: Heme oxygenase-1 posttranslational modifications in the brain of subjects with Alzheimer disease and mild cognitive impairment. Free Radic Biol Med 52: 2292-2301, 2012.

20. Kapitulnik J and Maines MD: Pleiotropic functions of biliverdin reductase: Cellular signaling and generation of cytoprotective and cytotoxic bilirubin. Trends Pharmacol Sci 30: 129-137, 2009.

21. Barone E, Di Domenico F, Mancuso C and Butterfield DA: The Janus face of the heme oxygenase/biliverdin reductase system in Alzheimer disease: It's time for reconciliation. Neurobiol Dis 62: 144-159, 2013.

22. Smith MA, Perry G and Pryor WA: Causes and consequences of oxidative stress in Alzheimer's disease. Free Radic Biol Med 32: 1049,2002

23. Barone E, Di Domenico F and Butterfield DA: Statins more than cholesterol lowering agents in Alzheimer disease: Their pleiotropic functions as potential therapeutic targets. Biochem Pharmacol 88: 605-616, 2014

24. Tudor C, Lerner-Marmarosh N, Engelborghs Y, Gibbs PE and Maines MD: Biliverdin reductase is a transporter of haem into the nucleus and is essential for regulation of HO-1 gene expression by haematin. Biochem J 413: 405-416, 2008.

25. Di Domenico F, Perluigi M and Barone E: Biliverdin Reductase-A correlates with inducible nitric oxide synthasein in atorvastatin treated aged canine brain. Neural Regen Res 8: 1925-1937, 2013.

26. Mancuso $\mathrm{C}$ and Barone E: The heme oxygenase/biliverdin reductase pathway in drug research and development. Curr Drug Metab 10: 579-594, 2009.

27. Lerner-Marmarosh N, Shen J, Torno MD, Kravets A, Hu Z and Maines MD: Human biliverdin reductase: A member of the insulin receptor substrate family with serine/threonine/tyrosine kinase activity. Proc Natl Acad Sci USA 102: 7109-7114, 2005.

28. Butterfield DA, Drake J, Pocernich C and Castegna A: Evidence of oxidative damage in Alzheimer's disease brain: Central role for amyloid beta-peptide. Trends Mol Med 7: 548-554, 2001.

29. Yan H, Li L and Tang XC: Treating senile dementia with traditional Chinese medicine. Clin Interv Aging 2: 201-208, 2007.

30. Bi M, Tong S, Zhang Z, Ma Q, Zhang S, Luo Z, Zhang Y, Li X and Wang D: Changes in cerebral glucose metabolism in patients with mild-to-moderate Alzheimer's disease: A pilot study with the Chinese herbal medicine fuzhisan. Neurosci Lett 501: 35-40, 2011.

31. Wu AG, Wong VK, Xu SW, Chan WK, Ng CI, Liu L and Law BY: Onjisaponin B derived from Radix Polygalae enhances autophagy and accelerates the degradation of mutant $\alpha$-synuclein and huntingtin in PC-12 cells. Int J Mol Sci 14: 22618-22641, 2013.

32. Han P, Han T, Peng W and Wang XR: Antidepressant-like effects of essential oil and asarone, a major essential oil component from the rhizome of Acorus tatarinowii. Pharm Biol 51: 589-594, 2013.

33. He JY, Zhu S, Goda Y, Cai SQ and Komatsu K: Quality evaluation of medicinally-used Codonopsis species and Codonopsis Radix based on the contents of pyrrolidine alkaloids, phenylpropanoid and polyacetylenes. J Nat Med 68: 326-339, 2014.

34. Zhang H, Zhang L and Liu Y: Studies on chemical components and pharmacological activities of Os Draconis (Longgu) and Ostreae Concha. Zhongguo Zhong Yao Za Zhi 36: 1839-1840, 2011 (In Chinese).

35. Xu Y, Song W, Zhou P, Li P and Li H: Morphological and microscopic characterization of five commonly-used testacean traditional Chinese medicines. Acta Pharm Sin B 5: 358-366, 2015.

36. Yang X, Zhou SL, Ma AC, Xu HT, Guan HS and Liu HB: Chemical profiles and identification of key compound caffeine in marine-derived traditional Chinese medicine Ostreae concha. Mar Drugs 10: 1180-1191, 2012.

37. Wu JJ, Sun WY, Hu SS, Zhang S and Wei W: A standardized extract from Paeonia lactiflora and Astragalus membranaceus induces apoptosis and inhibits the proliferation, migration and invasion of human hepatoma cell lines. Int J Oncol 43: 1643-1651, 2013. 